A. Pramesh Rao, G. Swarup and Gopal-Krishna, eds.

\title{
Preliminary Results of Galactic Radio Recombination Line Observations using the GMRT
}

\author{
N.G. Kantharia and D. Anish Roshi \\ National Centre for Radio Astrophysics, TIFR Pune, India
}

\begin{abstract}
A pilot project to observe recombination lines of hydrogen and carbon from Galactic HII regions near $20 \mathrm{~cm}(n=168)$ and $49 \mathrm{~cm}$ $(n=220)$ using the 30 -station correlator at the Giant Metre-wave Radio Telescope was undertaken. The preliminary results from observations of the HII regions W3 and S106 are presented here.
\end{abstract}

\section{Introduction}

Recombination lines (RLs) are a powerful probe of the physical conditions of ionized gas. We imaged W3 and S106 in continuum and searched for recombination line emission. The spectra are presented in this paper.

\section{Observations \& Preliminary Results}

The recombination line observations were conducted using the GMRT in OctoberNovember 1999 in the $20 \mathrm{~cm}$ and $49 \mathrm{~cm}$ bands. We detected $\mathrm{H} 168 \alpha$ and $\mathrm{C} 168 \alpha$ lines from W3A (Fig 1(a)); our 'calibration' lines which is an encouraging result. The $20 \mathrm{~cm}$ RLs towards W3A, which were detected earlier, originate in a PIR with $T_{e}=100 \mathrm{~K}, n_{e}=10 \mathrm{~cm}^{-3}$ and $\mathrm{EM}=390 \mathrm{~cm}^{-6} p c$ (Kantharia et al 1998). The expected strength of $\mathrm{H} 220 \alpha$ line, then, is $\sim 18 \mathrm{mJy}$. However there is no detection down to a $5 \sigma$ limit of $14 \mathrm{mJy}$. The electron density in the PIR could be larger than $10 \mathrm{~cm}^{-3}$ making the line undetectable at $49 \mathrm{~cm}$ due to continuum optical depth effects. However, we need confirmatory observations.

The spectra from S106 at $20 \mathrm{~cm}$ and $49 \mathrm{~cm}$ are shown in Fig 2. No clear signal is detected in the spectra.

The results of our pilot project of observing recombination lines towards Galactic HII regions are encouraging. We detected the narrow $\mathrm{H} 168 \alpha$ and $\mathrm{C} 168 \alpha$ lines from our 'calibration' source, W3A. Repeat GMRT observations are required for other frequencies and S106.

\section{References}

Kantharia, N.G., Anantharamaiah, K.R., Goss, W.M., 1998, ApJ, 504, 375. 

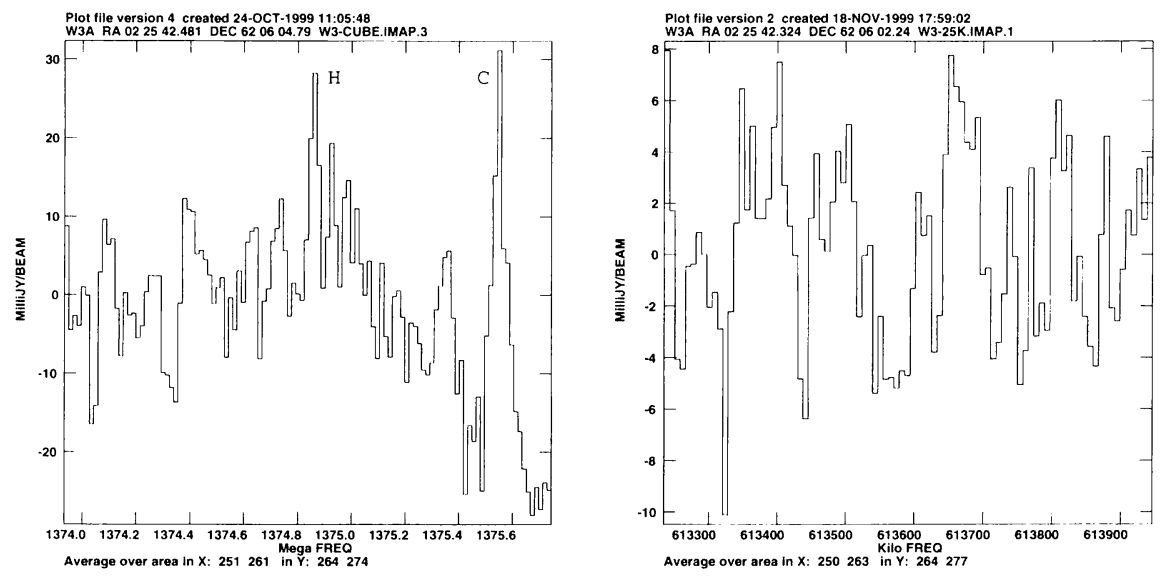

Figure 1. $168 \alpha$ and $220 \alpha$ spectra integrated over W3A. The $\mathrm{H}$ and $\mathrm{C}$ lines are seen at expected frequencies in $168 \alpha$ spectrum. No line is detected in the $220 \alpha$ spectrum. Both the spectra have an integration time $\sim 4$ hours.
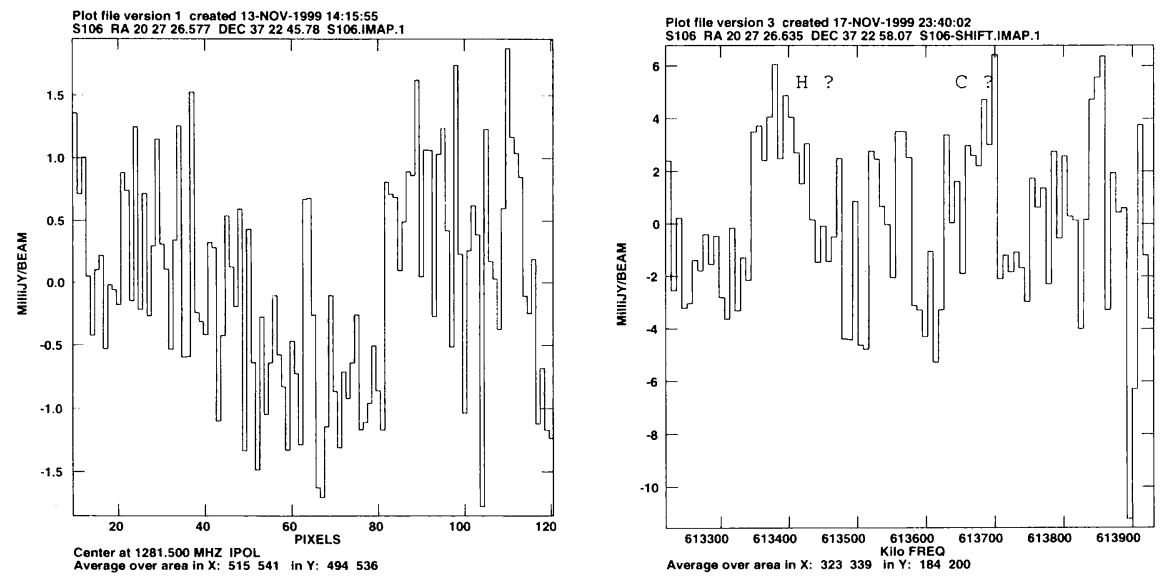

Figure 2. $168 \alpha$ and $220 \alpha$ spectra integrated over S106. No clear line detection. Note that the spectrum at $49 \mathrm{~cm}$ has suspect features at two frequencies. Both the spectra have an integration time $\sim 4$ hours. 